\section{Iniquidades sociais influenciam a qualidade e a diversidade da dieta de crianças brasileiras de 6 a 36 meses}

\author{
Social inequalities influence the quality and \\ diversity of diet in Brazilian children \\ 6 to 36 months of age
}

\author{
Desigualdades sociales influyen en la calidad y \\ diversidad de la dieta de los niños brasileños \\ con una edad de 6 a 36 meses
}

\author{
1 Universidade de Brasília, \\ Brasília, Brasil. \\ 2 Ministério da Saúde, \\ Brasília, Brasil. \\ 3 Universidade Federal de \\ Ciências da Saúde de Porto \\ Alegre, Porto Alegre, Brasil. \\ Correspondência \\ G. A. Bortolini \\ Al. dos Eucaliptos, Quadra 107 \\ Ed. das Américas, apto. 508, \\ Brasília, DF 71920-010, Brasil. \\ gisele.bortolini@gmail.com
}

\begin{abstract}
The objective of this study was to assess dietary patterns in Brazilian children and factors associated with better diet. The authors used data for 2,477 children 6 to 36 months of age from the Brazilian National Survey of Demographic and Health in 2006-2007. Diet was assessed and classified using a composite index. The results showed that $28.2 \%$ of the children received $a$ high-quality diet and $20 \%$ had a diversified diet. Children from socioeconomically underprivileged families or with serious food insecurity were approximately $40 \%$ less likely to have highquality diets. Children living in homes with food insecurity were $71 \%$ less likely to have diversified diets, and those whose mothers had limited education were $43 \%$ less likely. Children residing in the North of Brazil were less likely to have diversified and high-quality diets. The dietary quality of Brazilian children is inadequate, and social vulnerability is closely associated with this adverse dietary situation.
\end{abstract}

Food Consumption; Child Nutrition; Nutrition Programs and Policies; Nutritional Epidemiology
Gisele Ane Bortolini 1,2

Márcia Regina Vitolo 3

Muriel Bauermann Gubert 1

Leonor Maria Pacheco Santos 1

\section{Resumo}

Avaliar práticas alimentares de crianças brasileiras e os fatores associados à qualidade e à diversidade da dieta. Foram utilizados dados de 2.477 crianças com idade de 6 a 36 meses da Pesquisa Nacional de Demografia e Saúde realizada no Brasil em 2006/2007. As dietas foram avaliadas e classificadas por meio de um índice composto. Apresentaram dieta de alta qualidade $28,2 \%$ e dieta diversificada $20 \%$ das crianças. Crianças pertencentes às classes socioeconômicas menos privilegiadas e residentes em domicílio em situação de insegurança alimentar grave apresentaram, aproximadamente, $40 \%$ menos chances de ter dieta de alta qualidade. A chance de ter dieta diversificada foi $71 \%$ menor para crianças residentes em domicílio em situação de insegurança alimentar grave e 43\% menor se filhas de mães com baixa escolaridade. Crianças residentes na Região Norte do país apresentaram menos chances de ter dieta diversificada e dieta de alta qualidade. A qualidade da dieta de crianças brasileiras é inadequada, e a situação de vulnerabilidade social está fortemente associada a esse quadro alimentar desfavorável.

Consumo de Alimentos; Nutrição da Criança; Programas e Políticas de Nutrição e Alimentação; Epidemiologia Nutricional 


\section{Introdução}

As práticas alimentares inadequadas na infância têm impacto negativo nas condições de saúde e nutrição das crianças e, por consequência, no desenvolvimento infantil 1,2,3,4,5. No mundo, milhões de crianças menores de cinco anos, residentes em países em desenvolvimento, não atingem seu potencial de desenvolvimento, sendo a nutrição inadequada um dos fatores determinantes desse processo $1,2,3$, com consequências para toda a vida $6,7,8$.

Existem diversas formas de avaliar o consumo alimentar de crianças, incluindo índices que agregam componentes de avaliação da dieta e que permitem uma análise mais global da alimentação ${ }^{9}$. O índice de alimentação da criança 10 permite avaliar o consumo alimentar de crianças em pesquisas da série Demographic Health Surveys (DHS). Esse índice avalia as práticas alimentares das crianças e sumariza o tipo, a frequência e a qualidade em um único indicador, de acordo com as recomendações de alimentação saudável para cada faixa de idade 10 .

No Brasil, a II Pesquisa de Aleitamento Materno 11 , realizada em 2008, avaliou as práticas alimentares das crianças menores de 12 meses nas capitais brasileiras, e a Pesquisa Nacional de Demografia e Saúde (PNDS), que compõe as DHS 12, realizada em 2006, avaliou as práticas alimentares de crianças menores de cinco anos de todas as regiões do país. Porém, nenhum estudo, até o momento, utilizou dados dessas pesquisas para avaliar práticas alimentares das crianças brasileiras por meio de um indicador composto, como o proposto por Ruel \& Menon 10.

Sabe-se que, para o adequado delineamento de políticas públicas, é importante que os países avaliem e conheçam as práticas alimentares das crianças, assim como os possíveis fatores determinantes. Dessa forma, o objetivo deste estudo foi avaliar as práticas alimentares de crianças brasileiras com idade de 6 a 36 meses a partir de um índice de qualidade e de diversidade da dieta e seus possíveis fatores associados.

\section{Metodologia}

Foram utilizados dados nacionais oriundos da PNDS de 2006, que é realizada no Brasil a cada dez anos e faz parte da série DHS. A PNDS caracteriza-se por ser um estudo transversal que objetiva evidenciar as condições de saúde e nutrição da população feminina em idade fértil (15 a 49 anos) e dos seus filhos menores de cinco anos 13 . A pesquisa possui representatividade nacional para as cinco macrorregiões geográficas do país e para as zonas urbanas e rurais. Foram avaliados 14.617 domicílios, e, investigadas $15.575 \mathrm{mu}$ lheres e 4.957 crianças menores de cinco anos. Detalhes da amostragem e coleta de dados estão descritos em outras publicações 13 .

Para avaliação do consumo alimentar de crianças de 6 a 36 meses, foi utilizado questionário que abordava aspectos relacionados ao aleitamento materno e ao consumo de alimentos aplicado às mães de crianças menores de cinco anos. Foi utilizado inquérito de frequência alimentar na última semana para avaliar o consumo alimentar das crianças. Foram investigadas a frequência de consumo dos seguintes alimentos: cereais (arroz ou macarrão), feijões, tubérculos (batata, batata-baroa, mandioca, cará, inhame, abóbora), verduras de folhas, legumes (tomate, cenoura, beterraba, berinjela, chuchu e abobrinha), frutas, iogurte, pão, carnes (boi e porco), frango, peixe, suco natural, alimentos fritos, doces, biscoitos ou bolachas, salgadinhos em pacote, refrigerante e sucos artificiais 13 .

Para a classificação da dieta das crianças, foi adaptado, de acordo com as recomendações brasileiras e as variáveis existentes, o índice de alimentação da criança (child feeding index) elaborado por Ruel \& Menon 10. O escore prevê pontuação "0", para práticas potencialmente negativas, e "1" ou “2”, para práticas positivas. Práticas consideradas muito importantes, como aleitamento materno na idade de 6 a 12 meses ou consumir carne regularmente entre 12 e 36 meses, receberam escore " 2 ". As práticas foram consideradas negativas ou positivas com base nas recomendações nacionais de alimentação saudável 14. A Tabela 1 apresenta os componentes do índice e a respectiva pontuação. A partir do índice de alimentação da criança, foi possível classificar as dietas em baixa qualidade (percentil $\leq 25$ ), qualidade intermediária (percentil > P25 a percentil $<$ P75) e alta qualidade (percentil $\geq 75$ ).

As principais alterações realizadas em relação ao índice original foram: (a) as crianças foram agrupadas de acordo com as recomendações de número/tipo de refeições/dia preconizadas pelo Ministério da Saúde no Brasil: idade de 6 meses (aleitamento materno e uma refeição principal e duas refeições intermediárias), de 7 a 11 meses (aleitamento materno, duas refeições principais e duas refeições intermediárias) e de 12 a 36 meses (continuidade do aleitamento materno, três refeições principais e duas refeições intermediárias); (b) consumo de outro leite no dia anterior, em vez de utilização de mamadeira, pois a variável utilização de mamadeira apresentava 1.653 dados ausentes; (c) grupos de alimentos utilizados para considerar dieta diversificada foram: cereais ou tubérculos + feijões + hortaliças 
Variáveis e escore utilizados para criar o Índice de Alimentação da Criança Adaptado.

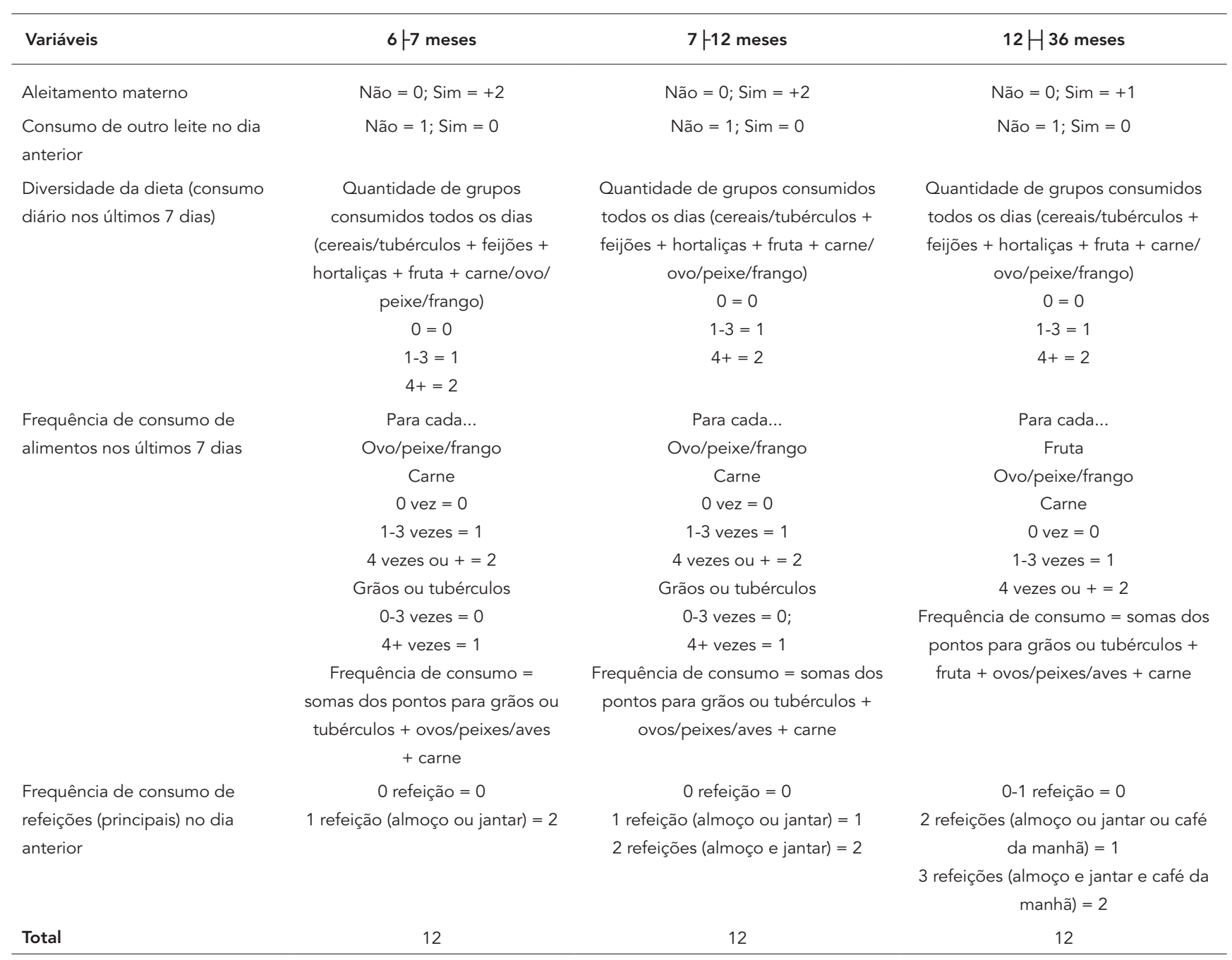

+ fruta + carne ou ovo ou peixe ou frango, pois são os grupos de alimentos recomendados no país; (d) consumo diário nos últimos 7 dias (em vez de consumo nas últimas 24 horas) dos grupos de alimentos pertencentes à análise da diversidade da dieta, uma vez que o instrumento utilizado no Brasil foi um inquérito de frequência de 7 dias; (e) substituição do grupo leite por fruta, uma vez que o consumo de leite é avaliado em outro componente, e a recomendação brasileira é de que as crianças sejam amamentadas até os dois anos e (f) considerar apenas a recomendação das refeições principais do dia descritas no item a, pois a informação de todas as refeições intermediárias, como, por exemplo, lanche da manhã, não está disponível no banco de dados.
O programa utilizado para as análises dos dados foi o Stata versão 11.0 (StataCorp LP, College Station, Estados Unidos). O plano de análise considerou o peso da amostra para crianças, o estrato e o conglomerado de residência. O número amostral apresentado refere-se ao número de crianças avaliadas na pesquisa; no entanto, todas as análises foram realizadas com amostra expandida, e os resultados são apresentados em percentuais. O banco de dados final contém informações de 2.477 crianças entre 6 e 36 meses, vivas no momento da entrevista e que moravam com a mãe. Para a composição do índice da criança, 2.344 crianças tinham informações de todos os componentes. 
Foi utilizada a regressão de Poisson bivariada para avaliar a associação das variáveis independentes com as variáveis dependentes e os resultados apresentados em razão de prevalência (RP) e respectivos intervalos de $95 \%$ de confiança (IC95\%). As variáveis dependentes utilizadas foram qualidade da dieta e diversidade da dieta, e as variáveis independentes investigadas neste estudo foram: região de residência, situação do domicílio (urbano ou rural), classificação socioeconômica, número de pessoas no domicílio, escolaridade materna, idade da mãe, trabalho materno, estado civil da mãe, grau de segurança e insegurança alimentar do domicílio e sexo e idade da criança. Para a análise ajustada, foi utilizada a regressão de Poisson multivariada, sendo que entraram no modelo apenas as variáveis associadas $(p<0,05)$ aos desfechos nas análises bivariadas.

A condição de insegurança alimentar domiciliar foi aferida pela Escala Brasileira de Insegurança Alimentar (EBIA) 15,16. A EBIA é composta de 14 itens que avaliam vários graus da experiência de insegurança alimentar e fome, sendo os domicílios categorizados em situação de segurança alimentar ou insegurança (leve, moderada ou grave). A classificação socioeconômica foi medida pelo poder aquisitivo e posse de bens, sendo as famílias classificadas em classes (A, maior poder de compra, até E, menor poder aquisitivo) 17 .

O estudo foi aprovado pelo Conselho de Ética em Pesquisa do Centro de Referência e Treinamento DST/AIDS da Secretaria de Estado de Saúde de São Paulo. A PNDS de 2006/2007 foi financiada pelo Ministério da Saúde.

\section{Resultados}

Aproximadamente, 35\% das mulheres apresentavam até quatro anos de estudo, e $43,4 \%$ pertenciam às classes socioeconômicas D e E, 18,5\% das famílias residiam em área rural, e apenas 54,1\% residiam em domicílios em situação de segurança alimentar e nutricional. Outras informações sobre outras características dos domicílios, das mães e das crianças encontram-se na Tabela 2.

É possível observar que a maioria das crianças menores de 12 meses de idade recebia leite materno, no entanto, o consumo de outros leites foi elevado nas duas faixas etárias. O consumo diário de frutas, hortaliças e carnes estava presente em menos que $50 \%$ das crianças; no entanto, a maioria das crianças consumira cereais ou tubérculos todos os dias, e mais da metade das crianças consumiram feijão diariamente. O consumo de, no mínimo, quatro grupos alimentares diariamente nos últimos sete dias, dos cinco gru- pos de alimentos investigados (diversidade da dieta), foi observado em aproximadamente $20 \%$ das crianças investigadas. Em torno de $70 \%$ das crianças entre 6 a 12 meses fizeram o desjejum e o jantar, no entanto, depois de 12 meses de idade, mais de $90 \%$ das crianças já faziam cada uma das três refeições principais (Tabela 3).

Com relação à dieta de baixa qualidade, observou-se prevalência de $47 \%$ em crianças de até 12 meses e $27,9 \%$ das maiores de 12 meses, sendo que $28,2 \%$ de todas as crianças apresentaram práticas alimentares classificadas como de alta qualidade (Tabela 4). A média do escore foi de $7,38 \pm 1,94$ para o grupo de crianças de 6 a 36 meses (dados não representados em tabela).

A Tabela 5 apresenta as análises dos possíveis fatores associados à alta qualidade e à diversidade da dieta resultantes das análises bivariadas e das regressões multivariadas. Aproximadamente, $40 \%$ das crianças residentes na Região Centro-oeste apresentaram dieta de alta qualidade, entretanto, na Região Norte, o percentual observado foi de apenas $21,9 \%$. Entre crianças filhas de mães com mais de 12 anos de escolaridade ou pertencentes às classes A e B, 38,9\% apresentam dieta de alta diversidade e, nos domicílios em situação de segurança alimentar, 30,9\% das crianças. Após análises ajustadas, é possível observar que as crianças residentes na Região Norte do país, residentes em domicílio em situação de insegurança alimentar grave e de baixo nível socioeconômico (D/E), apresentaram, respectivamente, chances de $28 \%, 40 \%$ e $39 \%$ menores de ter dieta de alta qualidade.

Entre as crianças filhas de mães com mais de 12 anos de escolaridade "e" pertencentes às classes A e B, " $44,2 \%$ e $41,1 \%$, respectivamente", apresentaram "dieta de alta qualidade" e, nos domicílios em situação de segurança alimentar, 30,9\% das crianças. Apenas 5,2\% das crianças residentes em domicílios em situação de insegurança alimentar grave tinham dieta diversificada, assim como apenas $10,3 \%$ das crianças residentes na Região Norte e 13,6\% das crianças residentes na área rural. Após análise ajustada, a chance de as crianças da Região Norte apresentarem dieta diversificada foi $45 \%$ menor do que as crianças da região de referência (Centro-oeste). Crianças filhas de mães com menos de quatro anos de escolaridade e residentes em domicílios em situação de insegurança alimentar grave apresentaram, respectivamente, chances de $43 \%$ e $71 \%$ menores de consumir dieta diversificada (Tabela 5). 
Tabela 2

Características do domicílio, das mães e das crianças com idade de 6 a 36 meses de idade. Brasil, 2006/2007.

\begin{tabular}{|c|c|c|}
\hline & $\mathrm{n}$ amostral * & $\begin{array}{l}\text { Todas ** } \\
\% \text { (IC95\%) }\end{array}$ \\
\hline \multicolumn{3}{|l|}{ Domicílio } \\
\hline \multicolumn{3}{|l|}{ Região geográfica } \\
\hline Norte & 529 & $10,6(9,1-12,3)$ \\
\hline Nordeste & 536 & $25,4(22,3-28,7)$ \\
\hline Sudeste & 461 & $42,6(38,1-47,2)$ \\
\hline Sul & 477 & $13,2(11,5-15,1)$ \\
\hline Centro-Oeste & 474 & $8,2(7,1-9,4)$ \\
\hline \multicolumn{3}{|l|}{ Local de residência } \\
\hline Urbana & 1.621 & $81,5(78,7-84,0)$ \\
\hline Rural & 856 & $18,5(16-21,3)$ \\
\hline \multicolumn{3}{|l|}{ Classificação socioeconômica } \\
\hline$A B$ & 300 & $15,1(12,2-18,5)$ \\
\hline C & 974 & $41,5(38,1-45,1)$ \\
\hline $\mathrm{DE}$ & 1.127 & $43,4(39,4-47,4)$ \\
\hline \multicolumn{3}{|l|}{ Nível de segurança alimentar } \\
\hline Segurança & 1.232 & $54,1(50-58,2)$ \\
\hline Insegurança leve & 635 & $26,3(22,8-30,2)$ \\
\hline Insegurança moderada & 285 & $11,7(9,6-14,3)$ \\
\hline Insegurança grave & 270 & $7,9(6,2-9,8)$ \\
\hline \multicolumn{3}{|c|}{ Número de pessoas no domićilio } \\
\hline $2-3$ & 637 & $34,3(30,9-37,9)$ \\
\hline $4-5$ & 1.146 & $48,3(44,6-52)$ \\
\hline$\geq 6$ & 694 & $17,4(14,9-20,1)$ \\
\hline \multicolumn{3}{|l|}{ Mães } \\
\hline \multicolumn{3}{|l|}{ Escolaridade (anos) } \\
\hline$\geq 12$ & 140 & $7,1(5,4-9,3)$ \\
\hline $5-11$ & 1.274 & $56,6(52,7-60,4)$ \\
\hline$<4$ & 987 & $36,3(32,6-40,2)$ \\
\hline \multicolumn{3}{|l|}{ Idade (anos) } \\
\hline$<20$ & 245 & $12,2(9,9-14,9)$ \\
\hline$\geq 20$ & 2.222 & $87,8(85,1-90,1)$ \\
\hline \multicolumn{3}{|l|}{ Estado civil } \\
\hline Solteira/Viúva/Divorciada & 377 & $14,5(12,1-17,1)$ \\
\hline Casada ou coabitação & 2.100 & $85,5(82,9-87,90$ \\
\hline \multicolumn{3}{|c|}{ Trabalha, além das atividades domésticas } \\
\hline Sim & 834 & $33,3(30,1-36,6)$ \\
\hline Não & 1643 & $66,7(63,4-69,9)$ \\
\hline \multicolumn{3}{|l|}{ Criança } \\
\hline \multicolumn{3}{|l|}{ Sexo } \\
\hline Menino & 1.321 & $54,7(51,1-58,2)$ \\
\hline Menina & 1.156 & $45,3(41,8-48,9)$ \\
\hline \multicolumn{3}{|l|}{ Idade (meses) } \\
\hline $6+7$ & 74 & $2,4(1,6-3,5)$ \\
\hline 7ㄴ-12 & 412 & $17,7(15,3-20,5)$ \\
\hline $12 \mathrm{H} 36$ & 1.991 & $79,9(77-82,5)$ \\
\hline
\end{tabular}

* Sem expansão, $n=2.477$ crianças de 6 a 36 meses;

** Análises realizadas com expansão da amostra. 
Tabela 3

Práticas alimentares em crianças brasileiras de 6 a 36 meses de idade. Brasil, 2006/2007.

\begin{tabular}{|c|c|c|c|}
\hline \multirow[t]{3}{*}{ Práticas alimentares } & \multirow[t]{3}{*}{$\mathrm{n}$ amostral * } & \multicolumn{2}{|c|}{ Idade da criança (meses) ** } \\
\hline & & $6+12$ & $12 Н 36$ \\
\hline & & $\%(I C 95 \%)$ & $\%(I C 95 \%)$ \\
\hline Aleitamento materno & 2.393 & $64,4(56,2-71,8)$ & $25,1(21,7-28,9)$ \\
\hline Consumo de outro leite & 2.445 & $76,5(70,0-82,0)$ & $85,2(82,4-87,6)$ \\
\hline Tipo de leite (leite de vaca) & 1.952 & $72,2(62,5-80,1)$ & $80,6(76,6-84,3)$ \\
\hline \multicolumn{4}{|l|}{ Consumo diário (últimos 7 dias) } \\
\hline Cereais e tubérculos & 2.452 & $69,5(63,2-75,1)$ & $80,0(76,5-83,1)$ \\
\hline Feijões & 2.442 & $54,8(47,6-61,9)$ & $66,0(62,4-69,5)$ \\
\hline Frutas & 2.454 & $46,4(38,5-54,5)$ & $44,1(39,2-49,1)$ \\
\hline Hortaliças & 2.439 & $33,5(26,7-41,1)$ & $23,6(20,2-27,2)$ \\
\hline Carnes & 2.438 & $18,9(13,4-26,1)$ & $31,5(27,5-35,8)$ \\
\hline Diversidade da dieta & 2.455 & $20,7(14,8-28,2)$ & $21,8(18,5-25,6)$ \\
\hline \multicolumn{4}{|c|}{ Frequência de consumo de alimentos nos últimos 7 dias (não comeu nenhum dia) } \\
\hline Ovo/Peixe/Frango & 2.453 & $35,1(27,9-43,1)$ & $6,9(5,2-9,1)$ \\
\hline Carne de boi ou de porco & 2.450 & $42,6(35,3-50,2)$ & $16,2(13,1-19,9)$ \\
\hline Frutas & 2.454 & $17,6(12,8-23,7)$ & $10,9(8,5-13,8)$ \\
\hline \multicolumn{4}{|c|}{ Frequência de consumo de refeições (principais) no dia anterior } \\
\hline Café da manhã & 2.476 & $70,3(63,0-76,7)$ & $92,2(89,6-94,1)$ \\
\hline Almoço & 2.476 & $88,6(83,2-92,4)$ & $94,4(92,3-96,0)$ \\
\hline Jantar & 2.476 & $71,0(64-77,2)$ & $91,0(88,5-93,0)$ \\
\hline
\end{tabular}

* Sem expansão, $n=2.477$ crianças de 6 a 36 meses;

** Análises realizadas com expansão da amostra.

Tabela 4

Descrição da classificação da dieta das crianças com idade de 6 a 36 meses de idade. Brasil, 2006/2007.

\begin{tabular}{lcccc}
\hline Idade (meses) & $\mathbf{n}$ amostral * & Baixa & $\begin{array}{c}\text { Qualidade da dieta (\%) ** } \\
\text { Intermediária }\end{array}$ & Alta \\
\hline 6 -12 & 459 & $47,0(39,1-55,1)$ & $23,9(18,1-30,9)$ & $29,1(22,0-37,3)$ \\
12 H 36 & 1.885 & $27,9(22,6-33,70$ & $44,2(39,2-49,3)$ & $27,9(24,3-31,9)$ \\
Todas & 2.344 & $31,6(27,1-26,6)$ & $40,2(36,1-44,5)$ & $28,2(24,7-31,9)$
\end{tabular}

* Sem expansão, $\mathrm{n}=2.477$ crianças de 6 a 36 meses;

** Análises realizadas com expansão da amostra. 
Fatores associados à alta qualidade da dieta e à diversidade da dieta em crianças com idade de 6 a 36 meses de idade. Brasil, 2006/2007.

\begin{tabular}{|c|c|c|c|c|c|c|}
\hline & \multicolumn{3}{|c|}{ Qualidade da dieta alta * } & \multicolumn{3}{|c|}{ Dieta diversificada * } \\
\hline & \multirow[t]{2}{*}{$\%$} & \multirow{2}{*}{$\begin{array}{c}\text { Poisson bivariada } \\
\text { RP (IC95\%) }\end{array}$} & \multirow{2}{*}{$\begin{array}{l}\text { Poisson ajustada } \\
\text { RP (IC95\%) }\end{array}$} & \multirow[t]{2}{*}{$\%$} & \multirow{2}{*}{$\begin{array}{l}\text { Poisson bivariada } \\
\text { RP (IC95\%) }\end{array}$} & \multirow{2}{*}{$\begin{array}{l}\text { Poisson ajustada } \\
\text { RP (IC95\%) }\end{array}$} \\
\hline & & & & & & \\
\hline \multicolumn{7}{|l|}{ Domicílio } \\
\hline \multicolumn{7}{|l|}{ Região geográfica } \\
\hline Norte & 21,9 & $0,57(0,42-0,76)$ & $0,72(0,52-0,98)$ & 10,3 & $0,37(0,25-0,55)$ & $0,55(0,37-0,83)$ \\
\hline Nordeste & 25,6 & $0,67(0,49-0,90)$ & $0,78(0,57-1,06)$ & 14,3 & $0,51(0,35-0,74)$ & $0,69(0,47-1,02)$ \\
\hline Sudeste & 27,5 & $0,72(0,52-0,99)$ & $0,72(0,51-1,00)$ & 25,1 & $0,90(0,64-1,27)$ & $0,88(0,62-1,25)$ \\
\hline Sul & 33,9 & $0,88(0,68-1,14)$ & $0,84(0,64-1,11)$ & 29,2 & $1,05(0,78-1,41)$ & $0,98(0,72-1,34)$ \\
\hline Centro-Oeste & 38,2 & 1,00 & & 27,7 & 1,00 & \\
\hline \multicolumn{7}{|l|}{ Local de residência } \\
\hline Urbana & 29,3 & 1,00 & 1,00 & 23,4 & 1,00 & 1,00 \\
\hline Rural & 22,8 & $0,77(0,60-1,00)$ & $0,87(0,67-1,13)$ & 13,6 & $0,58(0,40-0,83)$ & $0,74(0,50-1,11)$ \\
\hline \multicolumn{7}{|l|}{ Classificação socioeconômica } \\
\hline$A B$ & 41,1 & 1,00 & 1,00 & 30,2 & 1,00 & 1,00 \\
\hline C & 29,1 & $0,72(0,55-0,95)$ & $0,74(0,51-1,08)$ & 28,5 & $0,94(0,66-1,33)$ & $1,35(0,87-2,10)$ \\
\hline DE & 28,5 & $0,55(0,40-0,75)$ & $0,61(0,38-0,97)$ & 12,5 & $0,41(0,27-0,63)$ & $0,91(0,49-1,68)$ \\
\hline \multicolumn{7}{|l|}{ Nível de segurança alimentar } \\
\hline Segurança & 30,9 & 1,00 & 1,00 & 28,4 & 1,00 & 1,00 \\
\hline Insegurança leve & 26,9 & $0,87(0,64-1,17)$ & $1,01(0,74-1,38)$ & 17,8 & $0,62(0,45-0,86)$ & $0,73(0,51-1,04)$ \\
\hline Insegurança moderada & 27,4 & $0,88(0,58-1,34)$ & $1,11(0,74-1,38)$ & 9,4 & $0,32(0,18-0,59)$ & $0,47(0,25-0,89)$ \\
\hline Insegurança grave & 13,9 & $0,45(0,28-0,70)$ & $0,60(0,37-0,95)$ & 5,2 & $0,18(0,09-0,36)$ & $0,29(0,14-0,63)$ \\
\hline \multicolumn{7}{|c|}{ Número de pessoas no domićílio } \\
\hline $2-3$ & 26,3 & $0,93(0,69-1,25)$ & & 23,8 & $1,37(0,91-2,06)$ & \\
\hline $4-5$ & 29,4 & $1,04(0,77-1,39)$ & & 21,6 & $1,24(0,84-1,84)$ & \\
\hline$\geq 6$ & 28,3 & 1,00 & & 17,3 & 1,00 & \\
\hline \multicolumn{7}{|l|}{ Mães } \\
\hline \multicolumn{7}{|l|}{ Escolaridade (anos) } \\
\hline$\geq 12$ & 44,2 & 1,00 & 1,00 & 38,9 & 1,00 & 1,00 \\
\hline $5-11$ & 28,9 & $0,63(0,39-0,87)$ & $0,87(0,52-1,45)$ & 23,6 & $0,52(0,35-0,76)$ & $0,63(0,38-1,03)$ \\
\hline$<4$ & 25,9 & $0,58(0,39-0,87)$ & $0,94(0,54-1,63)$ & 16,0 & $0,39(0,25-0,61)$ & $0,57(0,33-0,99)$ \\
\hline \multicolumn{7}{|l|}{ Idade (anos) } \\
\hline$<20$ & 25,0 & $0,87(0,60-1,26)$ & & 21,3 & $1,10(0,70-1,72)$ & \\
\hline$\geq 20$ & 28,5 & 1,00 & & 23,5 & 1,00 & \\
\hline \multicolumn{7}{|l|}{ Estado civil } \\
\hline Solteira/Viúva/Divorciada & 26,3 & $0,92(0,65-1,30)$ & & 22,5 & $0,70(0,45-1,09)$ & $0,87(0,55-1,38)$ \\
\hline Casada ou coabitação & 28,4 & 1,00 & & 15,9 & 1,00 & 1,00 \\
\hline \multicolumn{7}{|l|}{ Trabalha, além das atividades } \\
\hline \multicolumn{7}{|l|}{ domésticas } \\
\hline Sim & 31,1 & 1,00 & 1,00 & 25,8 & 1,00 & \\
\hline Não & 26,7 & $0,85(0,68-1,07)$ & $0,95(0,75-1,19)$ & 19,4 & $0,75(0,57-0,98)$ & \\
\hline \multicolumn{7}{|l|}{ Criança } \\
\hline \multicolumn{7}{|l|}{ Sexo } \\
\hline Menino & 27,5 & $0,95(0,76-1,18)$ & & 21,6 & $1,00(0,69-1,45)$ & \\
\hline Menina & 28,9 & 1,00 & & 21,5 & 1,00 & \\
\hline Idade (meses) & & & & & & \\
\hline 6 나-12 & 29,1 & $1,03(0,79-1,36)$ & & 20,7 & $0,94(0,66-1,36)$ & \\
\hline $12 \mathrm{H} 36$ & 27,9 & 1,00 & & 21,8 & 1,00 & \\
\hline
\end{tabular}

IC95\%: intervalo de 95\% de confiança; RP: razão de prevalência.

* Análises realizadas com expansão da amostra. 


\section{Discussão}

A qualidade da dieta das crianças brasileiras de 6 a 36 meses está aquém do recomendado, sendo que a maioria das crianças apresentou dietas de qualidade intermediária ou baixa. A média do escore observado para as crianças brasileiras é muito próxima da observada para crianças residentes na Bolívia, Colômbia, Guatemala, Nicarágua e Peru, na década de 1990 10, e mais recentemente em Madagascar 18. Nesses países, melhores práticas alimentares estão associadas ao maior crescimento infantil, principalmente em crianças residentes em domicílio de baixa condição socioeconômica 10,18.

A proporção de crianças que foram classificadas como tendo dieta de alta qualidade não variou em função da idade, no entanto, maior proporção de crianças com idade de 6 a 12 meses apresentou dieta de baixa qualidade. Isso pode ser explicado pelos componentes do índice, uma vez que, apesar de haver descontinuidade do aleitamento materno após o primeiro ano de vida e menor consumo de hortaliças, a maioria das crianças maiores passa a fazer as três refeições principais e a consumir mais cereais/tubérculos, feijões, carnes e ovos nessa idade, prática não necessariamente adotada na introdução da alimentação complementar, apesar de recomendada para as crianças menores. Outro fator que pode explicar esses achados é o fato de que ainda existem muitos mitos e tabus acerca do que pode ou não ser ofertado à criança nas primeiras papas, fazendo com que as crianças de 6 a 12 meses tenham a introdução de alguns grupos alimentares postergada, como é o caso de ovos e carnes 19. A discussão sobre o consumo dos grupos alimentares que compõem o índice, em crianças brasileiras menores de cinco anos, foi realizada em estudos anteriores 20,21 .

É possível observar que existe a descontinuidade da amamentação após o primeiro ano de vida, no entanto, muitos foram os avanços em relação a essa prática no primeiro ano de vida no Brasil. A duração mediana da amamentação aumentou de 2,5 para 11,3 meses, e a prevalência da amamentação exclusiva em crianças brasileiras menores de seis meses passou de $3,1 \%$ para $41 \%$ no período de 1975 a 2008 22. De qualquer forma, evidencia-se a necessidade de ações de promoção, proteção e apoio ao aleitamento materno principalmente para mães com crianças maiores, pois a tendência no país é a interrupção da amamentação antes do primeiro ano de vida e o uso do leite de vaca 21 .

É interessante observar que apesar de a maioria das crianças menores de doze meses receberem a refeição almoço, no entanto, parte delas não recebe a refeição jantar, que, no país, é recomendado desde o sétimo mês. Além disso, a maioria das crianças já recebia uma refeição no desjejum, apesar de ser recomendado a partir dos 12 meses 14. É importante a realização de outros estudos que permitam avaliar a qualidade de cada uma das refeições, pois já foi demonstrado que crianças residentes em domicílios em situação de insegurança alimentar e de baixa renda tendem a receber a refeição jantar em menor frequência e com menos opções de variações 23 . Também é comum a elevada frequência de consumo de refeições lácteas em substituição a refeições baseadas em outros alimentos no país 24 .

Outro componente do índice que merece destaque é o da diversidade da dieta, pois somente cerca de $20 \%$ das crianças brasileiras consumiram quatro ou mais grupos de alimentos todos os dias, na semana anterior à pesquisa. Dessa forma, é possível inferir que a dieta das crianças brasileiras é, em geral, monótona e pode contribuir para o agravamento das principais deficiências nutricionais ainda existentes no país 12 . O indicador de diversidade da dieta tem sido muito utilizado como forma de avaliar a qualidade da dieta, a ingestão de micronutrientes e associação com desnutrição e carências nutricionais 4,25,26. Recentemente, a Organização Mundial da Saúde (OMS) passou a recomendar a avaliação da diversidade da dieta como um dos indicadores da alimentação complementar nos países 27 . O indicador de diversidade da dieta proposto pela OMS estabelece, como ponto e corte, o consumo de, pelo menos, quatro grupos de alimentos dos sete recomendados. No entanto, esse indicador foi proposto em 2010, após a realização da última PNDS no Brasil, e, por isso, a proposta da OMS não pode ser utilizada no presente estudo. Optou-se por utilizar, como indicador de dieta diversificada, a combinação dos grupos alimentares recomendados no país 14 .

A análise dos fatores associados à alta qualidade e à diversidade da dieta mostrou que a região de residência da criança, a classe socioeconômica, o grau de insegurança alimentar do domicílio e a escolaridade materna determinam se as crianças têm acesso ou não a uma dieta mais saudável. Dessa forma, é possível observar que, no período de realização do estudo, o consumo alimentar das crianças variou em função das condições sociais das famílias.

Nesse estudo, crianças filhas de mães com menos de quatro anos de estudo apresentaram menor chance de ter dieta diversificada. Corroborando com os resultados desse estudo, o nível de escolaridade materna e de trabalho materno foi identificado como determinante das práticas alimentares em estudo que avaliou dados de 
DHS de 20 países provenientes da África, Ásia e América Latina. Nesse mesmo estudo, os autores recomendam que mães de maior escolaridade e que trabalham fora devem receber ações que estimulem a continuidade da amamentação, para evitar o uso de outros leites, e as mães de menor escolaridade devem ser foco de ações que fomentem a diversidade da alimentação complementar 28 .

O grau de escolaridade materna também influencia a oferta de alimentos não saudáveis para as crianças. No Brasil, crianças residentes nas capitais, filhas de mães sem escolaridade apresentam chances duas a três vezes maiores de consumir sucos processados, refrigerantes e açúcar ou mel do que as filhas de mães com maior escolaridade 29 . Como alternativa para essa situação, já foi demonstrado que aconselhamento nutricional nos primeiros anos de vida contribui para as melhores práticas alimentares, mesmo em populações de baixa condição socioeconômica 30 .

A avaliação da segurança alimentar por meio da EBIA nos domicílios permite avaliar o acesso quantitativo e qualitativo aos alimentos e é um importante indicador para o monitoramento de iniquidades 31,32 . Nesse estudo, crianças que residiam em domicílios em situação de insegurança alimentar grave apresentaram menor chance de ter dieta de alta qualidade e menor chance de ter dieta diversificada. No Brasil, a maioria dos domicílios em situação de insegurança alimentar concentra-se nas regiões Norte e Nordeste 32 . No presente estudo, também se evidenciou que as crianças residentes na Região Norte apresentam menor chance de ter uma dieta de alta qualidade e diversificada. No país, as mais elevadas prevalências de baixo peso e baixa estatura concentram-se nessa região, provavelmente como reflexo da situação das iniquidades sociais 12 .

Crianças pertencentes a famílias de classes sociais mais baixas apresentaram menor chance de ter dieta diversificada. Recentemente, foi demonstrado que a insegurança alimentar moderada ou grave seria reduzida em $59,5 \%$ no Nordeste com uma renda familiar per capita mínima de $\mathrm{R} \$ 175,00$ ao mês, sinalizando que o aumento da renda familiar dos mais pobres e a melhor focalização do Programa Bolsa Família (PBF) são essenciais para a diminuição da insegurança alimentar no país 33. Já foi demonstrado que o programa contribuiu com a redução de $17 \%$ na mortalidade infantil geral e com a redução de $65 \%$ nos casos de morte por desnutrição e de $53 \%$ nos casos de morte por diarreia 34 . Também já foi demonstrado que o tempo de permanência no Programa, ou seja, o maior período de exposição à transferência de renda e aos serviços de saúde tende a ampliar a possibilidade de melhorias no estado nutricional das crianças beneficiárias 35 .

Apesar de os dados terem sido coletados no ano de 2006/2007, essa é a pesquisa nacional mais atualizada que avaliou o consumo alimentar de crianças menores de cinco anos de forma representativa para todas as regiões do país. A próxima DHS a ser realizada no Brasil está prevista para ir a campo apenas em 2016/2017. Assim sendo, os resultados deste estudo reforçam as recomendações internacionais de que, para garantir a adequada nutrição e o desenvolvimento infantil, são necessárias políticas públicas universais para romper o ciclo de transmissão intergeracional da pobreza que mantém milhões de crianças e suas famílias residentes em países pobres em situação de iniquidade social. Portanto, além de intervenções de saúde e nutrição, são necessárias ações integradas que envolvam os diversos setores, como, por exemplo, a economia, a saúde, a educação, a assistência social e a agricultura, para garantir o acesso de todos à alimentação adequada e saudável 36,37,38.

Em conclusão, os resultados deste estudo mostraram que a dieta das crianças brasileiras de seis a trinta e seis meses é de qualidade intermediária ou baixa e não apresenta diversidade. Além disso, crianças pertencentes a famílias de baixa renda, filhas de mães com menor escolaridade e residentes em domicílio em situação de insegurança alimentar grave nas regiões mais carentes do país são mais vulneráveis e apresentam menor chance de ter uma dieta saudável. Assim, além de intervenções de saúde para promover a alimentação adequada e saudável, é de fundamental importância a universalização de medidas estruturantes para a redução das desigualdades sociais e assim oportunizar que as crianças cresçam e se desenvolvam de forma saudável. 


\section{Resumen}

Evaluar las prácticas alimentarias de los niños brasileños y los factores asociados con mejores prácticas dietéticas. Se utilizaron los datos de 2.477 niños de 6 a 36 meses de la Encuesta Nacional de Demografía y Salud, llevada a cabo en Brasil en 2006/7. Las dietas fueron evaluadas y calificadas por un índice compuesto. Presentaron una dieta de alta calidad el $28,2 \%$ y una dieta variada el $20 \%$ de los niños. Niños pertenecientes a clases socioeconómicas menos privilegiadas, y que viven en domicilios en situación de inseguridad alimentaria grave, presentaron aproximadamente un $40 \%$ menos de probabilidad de tener una dieta de alta calidad. La probabilidad de tener una dieta diversificada fue un $71 \%$ menor en los niños que viven en domicilios en situación de inseguridad alimentaria grave y un 43\% menor, si son hijas de madres con bajo nivel de escolaridad. Los niños que viven en el norte del país tenían menos probabilidad de tener una dieta diversificada y de alta calidad. La calidad de la dieta de los niños es inadecuada y la situación de vulnerabilidad social está asociada con el panorama alimentario desfavorable.

Consumo de Alimentos; Nutrición del Niño;

Programas y Políticas de Nutrición y Alimentación,

Epidemiología Nutricional

\section{Colaboradores}

G. A. Bortolini contribuiu com a concepção do estudo, análise e interpretação dos dados, redação do artigo e aprovação final da versão a ser publicada. M. R. Vitolo, M. B. Gubert e L. M. P. Santos contribuíram com a concepção do estudo, revisão crítica relevante do conteúdo intelectual e aprovação final da versão a ser publicada.

\section{Agradecimentos}

Ao Ministério da Saúde pelo financiamento da Pesquisa Nacional de Demografia e Saúde e disponibilização do banco de dados em plataforma pública.

\section{Referências}

1. Grantham-McGregor S, Cheung YB, Cueto S, Glewwe P, Richter L, Strupp B. Developmental potential in the first 5 years for children in developing countries. Lancet 2007; 369:60-70.

2. Walker SP, Wachs TD, Gardner JM, Lozoff B, Wasserman GA, Pollitt E, et al. Child development: risk factors for adverse outcomes in developing countries. Lancet 2007; 369:145-57.

3. Walker SP, Wachs TD, Grantham-McGregor S, Black MM, Nelson CA, Huffman SL, et al. Inequality in early childhood: risk and protective factors for early child development. Lancet 2011; 378:1325-38.
4. Arimond M, Ruel MT. Dietary diversity is associated with child nutritional status: evidence from 11 demographic and health surveys. J Nutr 2004; 134:2579-85.

5. Vitolo MR, Bortolini GA, Dal Bo Campagnolo P, Feldens CA. Effectiveness of a nutrition program in reducing symptoms of respiratory morbidity in children: a randomized field trial. Prev Med 2008; 47:384-8.

6. Kuzawa CW, Hallal PC, Adair L, Bhargava SK, Fall $\mathrm{CH}$, Lee N, et al. Birth weight, postnatal weight gain, and adult body composition in five low and middle income countries. Am J Hum Biol 2012; 24:5-13. 
7. Fall $\mathrm{CH}$, Borja JB, Osmond C, Richter L, Bhargava SK, Martorell R, et al. Infant-feeding patterns and cardiovascular risk factors in young adulthood: data from five cohorts in low- and middle-income countries. Int J Epidemiol 2011; 40:47-62.

8. Martorell R, Melgar P, Maluccio JA, Stein AD, Rivera JA. The nutrition intervention improved adult human capital and economic productivity. J Nutr 2010; 140:411-4.

9. Smithers LG, Golley RK, Brazionis L, Lynch JW. Characterizing whole diets of young children from developed countries and the association between diet and health: a systematic review. Nutr Rev 2011; 69:449-67.

10. Ruel MT, Menon P. Child feeding practices are associated with child nutritional status in Latin America: innovative uses of the demographic and health surveys. J Nutr 2002; 132:1180-7.

11. Ministério da Saúde. II Pesquisa de Prevalência de Aleitamento Materno nas Capitais Brasileiras e Distrito Federal. Brasília: Ministério da Saúde; 2009.

12. Ministério da Saúde. Pesquisa Nacional de Demografia e Saúde da Criança e da Mulher - PNDS 2006: dimensões do processo reprodutivo e da saúde da criança. Brasília: Ministério da Saúde/ Centro Brasileiro de Análise e Planejamento; 2009.

13. Ministério da Saúde. Pesquisa Nacional de Demografia e Saúde da Criança e da Mulher - PNDS 2006: aspectos metodológicos. Brasília: Ministério da Saúde/Centro Brasileiro de Análise e Planejamento; 2008.

14. Ministério da Saúde. Dez passos para uma alimentação saudável: guia alimentar para menores de dois anos. Um guia para o profissional da saúde na atenção básica. Brasília: Ministério da Saúde; 2010.

15. Perez-Escamilla R, Segall-Correa AM, Kurdian Maranha L, Sampaio MMF, Marin-Leon L, Panigassi G. An adapted version of the U.S. Department of Agriculture Food Insecurity module is a valid tool for assessing household food insecurity in Campinas, Brazil. J Nutr 2004; 134:1923-8.

16. Pérez-Escamilla R, Segall-Corrêa AM. Food insecurity measurement and indicators. Rev Nutr PUCCAMP 2008; 21 Suppl:15s-26s.

17. Associação Brasileira de Empresas de Pesquisa. Critério de classificação econômica Brasil. http:// www.abep.org/ novo/FileGenerate.ashx?id=251 (acessado em 19/Jun/2014).

18. Moursi MM, Treche S, Martin-Prevel Y, Maire B, Delpeuch F. Association of a summary index of child feeding with diet quality and growth of 6-23 months children in urban Madagascar. Eur J Clin Nutr 2009; 63:718-24.

19. Takushi SAM, Tanaka ACA, Gallo PR, Bresolin AMB. Perspectiva de alimentação infantil obtida com gestantes atendidas em centros de saúde na cidade de São Paulo. Rev Bras Saúde Matern Infant 2006; 6:115-25.

20. Bortolini GA, Gubert MB, Santos LM. Consumo alimentar entre crianças brasileiras com idade de 6 a 59 meses. Cad Saúde Pública 2012; 28:1759-71.
21. Bortolini GA, Vitolo MR, Gubert MB, Santos LM. Early cow's milk consumption among Brazilian children: results of a national survey. J Pediatr (Rio J.) 2013; 89:608-13.

22. Venancio SI, Saldiva SR, Monteiro CA. Tendência secular da amamentação no Brasil. Rev Saúde Pública 2013; 47:1205-8.

23. Appelhans BM, Waring ME, Schneider KL, Pagoto SL. Food preparation supplies predict children's family meal and home-prepared dinner consumption in low-income households. Appetite 2014; 76:1-8.

24. Palmeira PA, Santos SMC, Vianna RPT. Prática alimentar entre crianças menores de dois anos de idade residentes em municípios do semiárido do Estado da Paraíba, Brasil. Rev Nutr PUCCAMP 2011; 24:553-63.

25. Moursi MM, Arimond M, Dewey KG, Treche S, Ruel MT, Delpeuch F. Dietary diversity is a good predictor of the micronutrient density of the diet of 6- to 23-month-old children in Madagascar. J Nutr 2008; 138:2448-53.

26. Steyn NP, Nel J, Labadarios D, Maunder EM, Kruger HS. Which dietary diversity indicator is best to assess micronutrient adequacy in children 1 to $9 \mathrm{y}$ ? Nutrition 2014; 30:55-60.

27. World Health Organization. Indicators for assessing infant and young child feeding practices - Part 1: conclusions of a consensus meeting held 6-8 November 2007 in Washington D.C., USA. Washington DC: World Health Organization; 2008.

28. Yarnoff B, Allaire B, Detzel P. Mother, infant, and household factors associated with the type of food infants receive in developing countries. Front Pediatr 2014; 2:14.

29. Saldiva SR, Venancio SI, de Santana AC, da Silva Castro AL, Escuder MM, Giugliani ER. The consumption of unhealthy foods by Brazilian children is influenced by their mother's educational level. Nutr J 2014; 13:33.

30. Vitolo MR, Bortolini GA, Dal Bo Campagnolo P, Hoffman DJ. Maternal dietary counseling reduces consumption of energy-dense foods among infants: a randomized controlled trial. J Nutr Educ Behav 2012; 44:140-7.

31. Panigassi G, Segall-Correa AM, Marin-Leon L, Perez-Escamilla R, Sampaio MF, Maranha LK. Insegurança alimentar como indicador de iniquidade: análise de inquérito populacional. Cad Saúde Pública 2008; 24:2376-84.

32. Poblacion AP, Marín-León L, Segall-Corrêa AM, Silveira JA, Taddei JAAC. Insegurança alimentar em domicílios brasileiros com crianças menores de cinco anos. Cad Saúde Pública 2014; 30:1067-78.

33. Facchini LA, Nunes BP, Motta JVS, Tomasi E, Silva SM, Thumé E, et al. Insegurança alimentar no Nordeste e Sul do Brasil: magnitude, fatores associados e padrões de renda per capita para redução das iniquidades. Cad Saúde Pública 2014; 30:161174.

34. Rassela D, Aquino R, Santos CAT, Paes-Sousa R, Barreto ML. Effect of a conditional cash transfer programme on childhood mortality: a nationwide analysis of Brazilian municipalities. Lancet 2013; 382:57-64. 
35. Jaime PC, Vaz ACN, Nilson EAF, Fonseca JCG, Guadagnin SC, Silva SA, Sousa MF, et al. Desnutrição em crianças de até cinco anos do Programa Bolsa Família: análise transversal e painel longitudial de 2008 a 2012. Cadernos de Estudos - Desenvolvimento Social em Debate 2014; 17:49-63.

36. Engle PL, Black MM, Behrman JR, Cabral de Mello M, Gertler PJ, Kapiriri L, et al. Strategies to avoid the loss of developmental potential in more than 200 million children in the developing world. Lancet $2007 ; 369: 229-42$.
37. Engle PL, Fernald LC, Alderman H, Behrman J, O'Gara C, Yousafzai A, et al. Strategies for reducing inequalities and improving developmental outcomes for young children in low-income and middle-income countries. Lancet 2011; 378:1339 53.

38. Ruel MT, Alderman H. Nutrition-sensitive interventions and programmes: how can they help to accelerate progress in improving maternal and child nutrition? Lancet 2013; 382:536-51.

Recebido em 17/Out/2014

Versão final reapresentada em 26/Abr/2015

Aprovado em 11/Mai/2015 